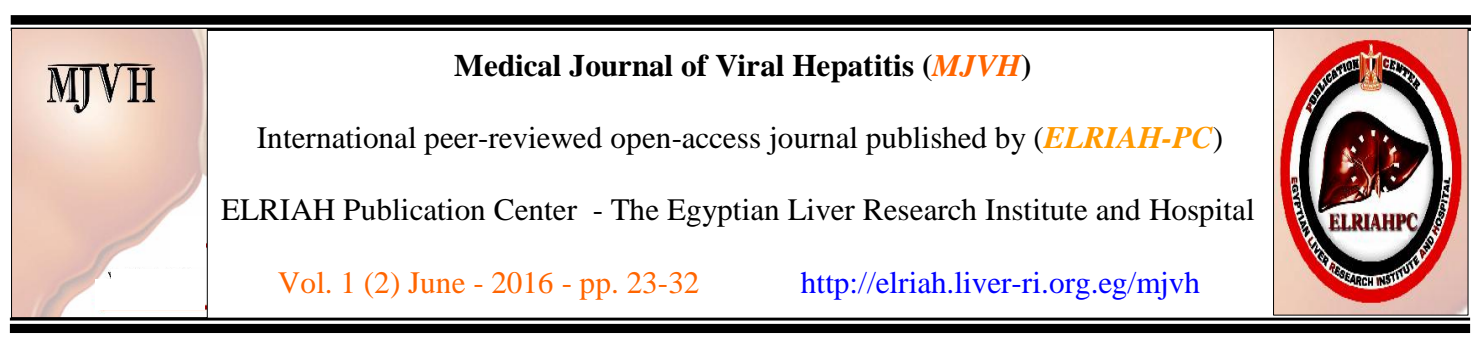

Original article

\title{
Transient elastography (FibroScan) is not useful in the diagnosis of schistosomal
}

\section{hepatic fibrosis}

\author{
Shiha, G. ${ }^{1,2 *}$, Samir, W. ${ }^{2}$, Soliman, R. ${ }^{2}$, Elbasiony $\mathrm{M}^{1,2}$, Ahmed, $\mathrm{N}^{2}$, Helmy, A. ${ }^{2,3}$ \\ ${ }^{I}$ Gastrohepatology Unit, Internal Medicine dept., Faculty of Medicine, Mansoura Univ., Egypt. \\ ${ }^{2}$ Egyptian Liver Research Institute and Hospital (ELRIAH), Mansoura, Egypt. \\ ${ }^{3}$ Tropical Medicine and Gastroenterology dept., Faculty of Medicine, Mansoura Univ., \\ Mansoura, Egypt \\ * E-mail: g_shiha@hotmail.com
}

Article History

Received: 23/3/2016

Revised: $1 / 7 / 2016$

Accepted: 12/8/2016

\section{Keywords:}

Non-invasive measures

liver fibrosis

bilharziasis

esophageal varices

Elastography

\begin{abstract}
:.
Transient Elastography (TE) is a widely-used noninvasive measure of liver stiffness. This study aimed to evaluate the diagnostic accuracy of TE in the diagnosis of schistosomal hepatic fibrosis (SHF). A total of 30 patients (Mean $\pm S D$ age $42.1 \pm 8.8$ years) with pure schistosomiasis were included. Abdominal ultrasound (US) and upper gastrointestinal endoscopy were performed to all patients to assess for signs of portal hypertension and the presence of varices as sequels of SHF. TE (FibroScan) was done to determine liver stiffness. A cutoff value of $\geq 10.1 \mathrm{Kpa}$ indicates advanced fibrosis (F3-4). Splenomegaly was detected in 27(90\%) patients and was moderate to marked $(\geq 15 \mathrm{~cm})$ in 19 (63.3\%). Esophageal and gastric varices were found in $25(83.3 \%)$ and $4(13.3 \%)$ cases respectively. TE was successful in all patients, and the mean $\pm S D$ liver stiffness was $9.4 \pm$ 5.5 Kpa (Range: 3.5-30 Kpa).F3-4 by FibroScan was detected in 8/25 $(32.0 \%)$ and $9 / 27(33.3 \%)$ of patients with EV and splenomegaly respectively. Similarly, F3-4 was absent in 4/5(80.0\%) and3/3 (100\%) of those without EV and splenomegaly advanced fibrosis respectively ( $p=0.71$ and $p=0.27$ respectively). To our knowledge, this study shows for the first time that TE is not useful in diagnosis of SHF and EV in patients with pure schistosomiasis. Whether this is applicable to other cases of prehepatic portal hypertension, such as portal vein thrombosis and congenital hepatic fibrosis, needs further investigation.
\end{abstract}

Abbreviations: EV; esophageal varices. HCV; hepatitis $C$ virus. IHA; indirect hemagglutination . Kpa; kilo Paskal. LB; liver biopsy. OR; odd ratio. PHT; portal hypertension. SHF; schistosomal hepatic fibrosis. TE; transient elastography. US; ultrasonography.

\section{Introduction}

Schistosomiasis currently affects more than 250 million people per year worldwide and it is estimated that 20 million people suffer severe morbidity due to schistosomiasis globally [1]. The three major schistosome species known to infect 
humans are Schistosoma hemat-obium (endemic in Africa and the eastern Mediterranean), Schistosomamansoni (endemic in Africa, the Middle East, the Caribbean and South America) and Schistosomajaponicum (endemic mainly in China, Japan and the Philippines) [2]. According to the largest survey conducted in Egypt, the prevalence of $S$. hematobium and S. mansoni infections is about $7.8 \%$ and $36.4 \%$ in Upper Egypt and Lower Egypt respectively [3]. S. hematobium, mainly affects the urinary tract, while $S$. mansoni and $S$. japonicum cause gastrointestinal and liver morbidities secondary to the passage of schistosome eggs from the lumen of the mesentric veins into adjacent tissues resulting in marked schistosomal hepatic fibrosis (SHF) known as pipestem fibrosis or Symmer's fibrosis. This subsequently leads to portal hypertension (PHT) and hepaticencephalopathy with their sequelae [2]. Despite availability and efficacy of praziquantel therapy, liver fibrosis, which is the most serious sequelae of chronic schistosomiasis develops in up to $20 \%$ of infected individuals [4]. The prognosis and management of advanced hepatic schistosomasis depends largely on the degree of liver fibrosis [5]. Despite being the gold standard in assessing hepatic fibrosis, use of liver biopsy (LB) is limited by poor patients' compliance, its associated morbidity, sampling error, poor intra- and inter-observer concordance, and by being unsuitable for dynamic surveillance [6,7]. Ultrasonography (US) has extensively been used to assess hepatic morbidity in patients with chronic schistosomiasis, but the associated inter-observer variations and the recent finding of only moderate correlation between US and LB findings makes US use in assessing HS-PPF inaccurate especially in non-severe case [8]. Moreover, only few studies have used serum biomarkers to assess the stage of hepatic fibrosis or esophageal varices (EV) in patients with schistosomiasis were reliable and sensitive markers for differentiating significant hepatic fibrosis in patients with advanced schistosomiasis japonica [9-11]. Transient elastography (TE; FibroScan) is a noninvasive, reproducible, user-friendly, and a well-established technique used in the initial assessment and follow up of hepatic fibrosis and cirrhosis [7,12]. The performance of TE in staging hepatic fibrosis was tested in a subgroup of Egyptian patients with positive schistosomiasis serology, but they were all coninfected with hepatitis $\mathrm{C}$ virus (HCV) [13]. However, it is well known that concurrent $\mathrm{HCV}$ and schistosomiasis infections result in an advanced and more severe liver disease than that observed with either disease alone [14]. To our knowledge, no previous study has used TE (FibroScan)as a non-invasive tool in the assessment of hepatic fibrosis in patients with pure chronic schistose-miasis, which is the aim of the present study.

\section{Patients and Methods}

\subsection{Patients}

A total of 30 Egyptian patients with mean \pm SD age of $42.1 \pm 8.8$ (range: $18-60$ ) years, with the diagnosis of pure Schistosoma mansoni were enrolled in the study. All patients were positive for indirect hemaggultination assay (IHA) for anti-schistosoma antibody with a titer 
$<1 / 160$ and all have already received praziquantil therapy for schistosomiasis.

\subsection{Exclusion criteria}

Patients with any of the following conditions or characteristics were excluded from participation: Age $<18$ or $>60$ years; active alcohol consumption; pre-existing psychiatric condition, pregnant or breast feeding women; significant clinical comorbidities; patients with co-existent liver disease, e.g. chronic hepatitis $\mathrm{C}$, chronic hepatitis B, or autoimmune liver diseases; patients who were currently on anti-viral therapy or who had completed anti-viral therapy and those who had ascites or focal lesions by abdominal US.

\subsection{Approval and consent}

This study was performed in the Egyptian Liver Research Institute and Hospital (ELRIAH), Sherbin, Dakahliya, Egypt between December 2013 and December 2014. The study was approved by the local Ethics Committee and the institution's review board, conducted in accordance with the Declaration of Helsinki and the International Conference on Harmonization guidelines, and each participant gave an informed written consent before being enrolled in the study and before performing the upper endoscopy.

\subsection{Clinical and laboratory evaluation}

The patients were subjected to thorough history taking, clinical examination, routine pre-treatment laboratory work-up including complete blood count, international normalized ratio (INR), and serum aspartate aminotransferase (AST), alanine aminotransferase (ALT), albumin, bilirubin, ferritin, and ceruloplasmin. Common terminology criteria Version 4.0 was used to grade the abnormal lab values
[15]. Serum samples from all patients were also tested for serological tests including anti-HCV antibodies, $\mathrm{HBsAg}$; $\mathrm{HBcAb}$; anti-nuclear antibody (ANA); anti-smooth muscle antibody (anti-SMA), anti-liver kidney microsomal antibody (anti-LKM), and pregnancy test for female subjects of child bearing potential. Antischistosomal antibodies were tested by the indirect hemagglutination (IHA) test. Previous exposure to Schistosoma was identified by a history of previous contact with canal water and/or receiving antibilharzial therapy with a positive serology titer $\geq 1 / 160$.

\subsection{Abdominal US and endoscopy}

Abdominal US was performed for all subjects using a Toshiba real time scanning device "AplioM" with a convex probe" to detect the presence of liver fibrosis and signs of portal hypertension (i.e., dilated portal vein $>14 \mathrm{~mm}$, dilated splenic vein $>9$ $\mathrm{mm}$, presence of splenic hilar collaterals and splenomegaly $>13 \mathrm{~cm}$ ). Also, an upper gastrointestinal endoscopy was performed to all patients to assess for signs of PHT and the presence of EV as sequels of SHF.

\subsection{FibroScan}

TE (FibroScan) was done to determine liver stiffness using the ultrasound TE fibroscan device (Echosens, Paris, France), which consists of a $5-\mathrm{MHz}$ ultrasound transducer probe mounted on the axis of a vibrator. Liver stiffness was measured on the same day as LB. TE measures liver stiffness in a volume that approximates a cylinder $1 \mathrm{~cm}$ wide and 4 $\mathrm{cm}$ long, between 25 and $65 \mathrm{~mm}$ below the skin surface, and the technique was performed according to that described in previous studies [16]. A cutoff value of $\geq 10.1 \mathrm{Kpa}$ indicates advanced fibrosis (F3- 
F4).The classification used for grading liver stiffness by TE (FibroScan) was a modification from that described by Castera et al. [17]. F0-F1: 6-7 kPa, F2: 7.1$9.4 \mathrm{kPa}, \mathrm{F} 3:$ 9.5-12.4 kPa and F4: $>12.5 \mathrm{kPa}$.

\subsection{Statistical analysis}

Data were collected in a pre-formed data collection form prior to entery in a Microsoft Excel Sheet and then were transferred to SPSS (Statistical Package for the Social Science; SPSS Inc., Chicago, IL, USA) version 15 for Microsoft Windows for analysis. The quantitative data were described with mean and standard deviation (SD) and compared by the Student's t-test.
Qualitative variables were described by number and percent. They were compared by the chi-squared or Fischer's exact test, when appropriate. In all tests, a $\mathrm{p}$ value $<0.05$ was considered significant.

\section{Results}

A total of 30 patients were included in this study. Their mean $\pm \mathrm{SD}$ age and body mass index (BMI) of all patients were $42.1 \pm 8.8$ years and $27.0 \pm 4.7 \mathrm{~kg} / \mathrm{m}^{2}$ respectively, and $25(83.3 \%)$ of them were males. Characteristics of patients are listed in tab. (1).

Table (1) Patients' demographics and baseline characteristics $(n=30)$.

\begin{tabular}{|c|c|}
\hline Variable & Result \\
\hline \multicolumn{2}{|l|}{ Age } \\
\hline$-<40$ years & $9(30.0)$ \\
\hline$-\geq 40$ years & $21(70.0)$ \\
\hline Years & $42.1 \pm 8.8(20-55)$ \\
\hline \multicolumn{2}{|l|}{ Sex } \\
\hline - Male & $25(83.3)$ \\
\hline - Female & $5(16.7)$ \\
\hline \multicolumn{2}{|l|}{ Body mass index } \\
\hline$-\leq 25 \mathrm{Kg} / \mathrm{m}^{2}$ & $18(60.0)$ \\
\hline$->25 \mathrm{Kg} / \mathrm{m}^{2}$ & $12(40.0)$ \\
\hline$-\mathrm{K} / \mathrm{m}^{2}$ & $27.0 \pm 4.7(19-38)$ \\
\hline Alanine transaminase IU/L & $41.0 \pm 33.9$ \\
\hline Aspartate transaminase IU/L & $39.0 \pm 29.6$ \\
\hline Serum albumin $\mathrm{gm} / \mathrm{dL}$ & $4.1 \pm 0.5$ \\
\hline Serum bilirubin $\mathrm{mg} / \mathrm{dL}$ & $1.0 \pm 0.3$ \\
\hline Platelets $\times 10^{3}$ & $148.4 \pm 76.1$ \\
\hline FibroScan Kpa & $9.4 \pm 5.5(3.5-30)$ \\
\hline \multicolumn{2}{|l|}{ Splenomegaly* } \\
\hline - Marked & $8(26.7)$ \\
\hline - Moderate & $7(23.3)$ \\
\hline - Mild & $12(40.0)$ \\
\hline$-\mathrm{No}$ & $3(10.0)$ \\
\hline Esophageal varices $* *$ Yes & $25(83.3)$ \\
\hline Gastric varices $^{\#}$ Yes & $4(13.3)$ \\
\hline \multicolumn{2}{|l|}{ Fibrosis stage ${ }^{* * *}$} \\
\hline$-\mathrm{F} 0-2$ & $22(73.3)$ \\
\hline$-\mathrm{F} 3-4$ & $8(26.7)$ \\
\hline
\end{tabular}

Data expressed as mean $\pm S D$ (range) or $n(\%)$ as appropriate. * by US scan.** by endoscopy. \#; all 4 patients with gastric varices had esophageal varices, i.e., included in the 25 patients. $n$; number. SD; standard deviation. *** by FibroScan.

Using US Scan, splenomegaly was detected in $27(90 \%)$ patients and was described as moderate to marked $(\geq 15 \mathrm{~cm})$ in $19(63.3 \%)$. EV were found during 
Upper Endoscopy in $25(83.3 \%)$ and 4/25 $(16.0 \%)$ patients had gastric varices together with the EV. FibroScan (TE) was successful in all patients and the mean \pm SD liver stiffness in all patients $(n=30)$ was $9.4 \pm 5.5 \mathrm{Kpa}$ (Range: $3.5-30 \mathrm{Kpa}$ ).Using FibroScan and a cutoff value of $10.1 \mathrm{Kpa}$, F0-F2 was detected in $22(73.3 \%)$ patients and F3-4 was detected in the remaining 8 $(26.7 \%)$. EV and splenomegaly were detected in 18/25 (81.8\%) and 19/27 (86.4 $\%)$ of cases with non-advanced fibrosis, and in $7(87.5 \%)$ and $8(100 \%)$ of cases with advanced fibrosis respectively ( $\mathrm{p}=0.71$ and $\mathrm{p}=0.27$ respectively, tab. (2). It was identified by executing descriptive cross tabulation that $72.0 \%$ of variceal cases and $70.7 \%$ of splenomegaly cases were diagnosed by FibroScan as F0-2, tab. (2). Overall it was noticed that out of 27 patients who had splenomegaly, only 8 were identified as F3 and F4 by FibroScan. Among variceal group, also only 7 were identified as F3-4 out of a total of 25 patients. It was interesting to see patients who have normal spleen were classified as F0-2, however, for the varices group $(\mathrm{n}=25)$, a single patient who did not have disease by Endoscopy was identified as F3 by FibroScan.

Table (2) Cross tabulation of hepatic fibrosis inpatients with varicesand splenomegaly as measured by FibroScan.*

\begin{tabular}{lcccc}
\hline \multirow{2}{*}{ FibroScan } & \multicolumn{2}{c}{ Splenomegaly } & \multicolumn{2}{c}{ Varices } \\
\cline { 2 - 5 } & No & Yes & No & Yes \\
\hline F0-2 & $19(70.4)$ & $03(100.0)$ & $18(72.0 \%)$ & $04(80.0)$ \\
F3-4 & $08(29.6)$ & $0(0.0)$ & $07(28.0 \%)$ & $01(20.0)$ \\
Total & $27(100.0)$ & $03(100.0)$ & $25(100.0)$ & $05(100.0)$ \\
\hline Data expressed an $n(\%) *$ using a cutoff value of $>10.1 \quad$ Kpa indicates advanced fibrosis
\end{tabular}

(F3-F4).

As shown in tab. (3), the sensitivity, specificity, PPV, NPV and accuracy of fibroScan in detecting advanced fibrosis evidenced by presence of spenomegaly were $29.6 \%, 100 \%, 100 \%, 13.64$, and $36.7 \%$. Similarly, the corresponding performance characteristics of FibroScan in detecting advanced fibrosis evidenced by presence of varices are $28.0 \%, 80.0 \%$, $87.5 \%, 18.2 \%$, and $37.9 \%$ respectively. Descriptive analysis was performed for the laboratory parameters AST, ALT and Bilirubin. Abnormal results for AST and
ALT were compared with the Endoscopy, US and FibroScan findings. It was interesting to see only one patient had Grade II ALT results (clinically significant) whereas 7 patients had Grade I AST and ALT results. Grade I abnormality for bilirubin was also noted for 7 patients. There appears no specific pattern for abnormal laboratory results on FibroScan findings because out of these patients only one subject had F4 fibrosis stage, and the subject who had Grade II ALT results has F1 Fibrosis stage as summarized in tab. (4).

Table (3) Performance features of FibroScan in the detection of splenomegaly and varices cases.

\begin{tabular}{lcc}
\hline \multicolumn{1}{c}{ Performance test } & $\begin{array}{c}\text { Splenomegaly patients } \\
(\boldsymbol{n}=\mathbf{2 7})\end{array}$ & $\begin{array}{c}\text { Varices patients } \\
(\boldsymbol{n}=\mathbf{2 5})\end{array}$ \\
\hline Sensitivity & $29.6(13.8-50.2)$ & $28.0(12.1-49.4)$ \\
Specificity & $100(29.2-100.0)$ & $80.0(28.4-99.5)$ \\
Positive Predictive Value & $100(63.1-100.0)$ & $87.5(47.4-99.7)$ \\
\hline
\end{tabular}


Data expresses as \% and (confidence interval).

Table (4) Comparison of abnormal ALT and AST results with Endoscopy, US and Fibrosis stage

\begin{tabular}{ccccc}
\hline $\begin{array}{c}\boldsymbol{A S T} \boldsymbol{T} \\
\text { Grade }\end{array}$ & $\begin{array}{c}\boldsymbol{A L T} \\
\text { Grade* }\end{array}$ & $\begin{array}{c}\text { Varices } \\
\text { by Endoscopy }\end{array}$ & $\begin{array}{c}\text { Splenomegaly } \\
\text { by US }\end{array}$ & Fibrosis Stage \\
\hline Grade I & Grade II & EV & Mild & F1 \\
Grade I & Grade I & EVs & Marked with PV $=22 \mathrm{~mm}$ & F1 \\
Grade I & Grade I & No EVs & Moderate with PV >13 mm & F2 \\
Normal & Grade I & No EVs & Normal Spleen & F1 \\
Grade I & Grade I & EVs & Mild & F1 \\
Grade I & Grade I & Fundal varices & Mild & F3 \\
Grade I & Grade I & No EVs & Mild & F0 \\
Grade I & Grade I & EV & Marked & F4 \\
\hline
\end{tabular}

$P V$; portal vein. ALT; alanine transaminase. AST; aspartate transaminase. EV; esophageal varices. According to Common terminology criteria Version 4.0i was used to grade the abnormal laboratory values

\section{Discussion}

This study shows for the first time that TE (FibroScan) is not useful in the diagnosis of SHF in patients with pure schistosomiasis. This is evidenced by the findings that almost one third of the patients who had proved by endoscopy to have varices by endoscopy were diagnosed by FibroScan as F0-2. Similarly, $>70 \%$ of patients who were diagnosed to have splenomegaly by US scan had F0-2 by FibroScan. These findings are consistent with the study conducted by Lebray et al in 2008, who showed that measuring liver stiffness is not a reliable marker of hepatic fibrosis in patients with congestive heart failure [18]. Simple noninvasive laboratory and US-based methods were utilized in the assessment of SHF. In a study by Wu et al, performed in 55 patients with advanced schistosomiasis japonica, utilizing LB as a gold-standard, HA and INR were reliable markers for differentiating significant liver fibrosis in patients with advanced schistosomiasis japonica. The authors concluded that this new simple index can easily predict significant SHF with a high degree of accuracy [10]. Platelets count/spleen diameter ratio has been found useful in predicting the presence of $\mathrm{EV}$ in patients with advanced hepatosplenic schistosomiasis in upper endoscopy [11]. Liver stiffness measurement using TE (FibroScan) and reference needle LB were both done in 229 Egyptian patients with chronic $\mathrm{HCV}$ infection, $29 \%$ of them had positive serology for Schistosoma mansoni infection. In this subgroup, the sensitivity of FibroScan in detecting fibrosis stages F2-3 was impaired. However, multivariate logistic regression showed that fibrosis stages F0-1 and F4 
were the most independent factors associated with the agreement between TE (FibroScan) and LB (ORs: 3.4 and 7.12 and $\mathrm{P}<0.001$ and $\mathrm{P}<0.001$ respectively) [13]. However, these cases had concurrent HCV infection and none of them had pure schistosomiasis. In agreement with our findings, Shiha and Zalata 2001, demonstrated that the co-existence of schistosomiasis does not interfere with application of the Knodell score in patients with chronic HCV infection [19]. Also, Abdel-Rahman et al 2013 showed that positive schistosomal serology has no effect on hepatic fibrosis staging in patients with chronic HCV infection [20].This has also been shown by earlier studies in Egyptian patients with both chronic HCV infection and positive Schistosoma serology, which showed a lack of enhancement of the $\mathrm{HCV}$-related pathology in the schistosomal patients [21]. FibroScan's sensitivity in detecting both varices and splenomegaly is low in our study (37.93\% and $36.66 \%$ respectively). Although literature suggests FibroScan is a good non-invasive method to predict EV presence and possible grading with high sensitivity, but the different results can be due to difference in patients as our study is in pure schistosomal patients and Saad tested HCV patients [22]. Descriptive results for the laboratory data suggest that there is no specific pattern for abnormal laboratory results on FibroScan findings. The grade I abnormalities detected for AST and ALT values are shown in table 5 and these abnormalities are compared with FibroScan stage. Only one of the patients with abnormal grade one lab finding had F4 fibrosis stage. Unlike patients with cirrhosis, those with
Schistosoma mansoni develop fibrosis that mainly involves the portal tracts (pipe-stem fibrosis), leading to a form of pre-hepatic PHT that leads to splenomegaly and varices with preserved liver functions. Advances or spread of the fibrosis process into the hepatic lobules occurs very late in the course of the disease and leads to liver cell failure. Therefore, LB and the METAVIR scoring system can't be considered as goldstandard in the evaluation of hepatic fibrosis in patients with schistosomiasis especially in early stages of the disease (prior to involvement of the whole lobule), which justifies the decision not to do LB for our patients. Indeed, the METAVIR, Ishak or Knoddle scoring systems were all developed and applied, for grading and staging inflammation and fibrosis respectively, in patients with parenchymal liver diseases of viral, metabolic, or alcoholic etiology, which involving the whole lobule rather the portal tracts as the case in schistos-omiasis and other causes of prehepatic PHT. In this study, we did not use LB as the gold-standard to assess SHF, and instead, we relied on endoscopy and ultrasound, in detecting varices and splenomegaly respectively as indicatives of advanced disease. Indeed, from 1991 to 2000, the WHO convened 3 Meetings on Ultrasonography in Schistosomiasis and establish a common grading system that include a qualitative assessment of liver parenchyma, height indexed measurements of portal branch wall thickening, portal vein enlargement, along with signs of portal hypertension (ascites, collateral vein enlargement) in a scoring matrix to define the presence of possible, probable, definite, or advanced schistosomiasis-related 
periportal fibrosis and/or portal hypertension [23-27]. One of the potential limitations of this study is the small number of subjects included. However, given the decreasing prevalence of schistosmiasis secondary to the wide-spread use of preziquantil together with other control measure, and the high prevalence of chronic $\mathrm{HCV}$ infection in the whole country, especially among patients with schistosomiasis makes the finding and the inclusion of 30 patients with pure schistosomiasis a satisfactory number. Indeed, the previous nationwide use of the trivalent antimonial compounds in treatment of schistosome-infected patients is considered responsible for the current epidemic of $\mathrm{HCV}$ infection in Egypt, and makes the finding of pure schistosomiasis cases a difficult task. It is recommended that the results of FibroScan should always be interpreted by a qualified clinician according to the clinical context, taking into account the patient demographics, disease etiology, and laboratory parameters. There is strong need to plan future studies with carefully defined different cutoff values for fibrosis staging. Longitudinal cohort studies can be effective prior to implementing the FibroScan results for the detection of SHF in patients with and without associated viral or other parenchymal liver disease.

\section{Conclusion}

In conclusion, the present study shows for the first time that, TE is not accurately indicative of patients' with pure Schistosoma mansoni clinical condition, and is especially not useful in the diagnosis of SHF and EV in such patients. However, these findings need to be confirmed in a larger group of patients. Whether this is applicable to other patients with other causes of pre-hepatic PHT, such as portal vein thrombosis and congenital hepatic fibrosis, needs further investigation.

\section{References}

[1] Chitsulo, L., Engels, D., Montresor, A., Savioli, L., (2000). The global status of schistosomiasis and its control. ActaTropica; 77: 41-51.

[2] Ross, A., Bartley, P., Sleigh, A., Olds, G., Li, Y., Williams, G., et al., (2002). Schistosomiasis. New Eng J. Med; 346: 1212-1220.

[3] El-Khoby, T., Galal, N., Fenwick, A., Barakat, R., El-Hawey, A., Nooman, Z., et al., (2000). The epidemiology of schistosomiasis in Egypt: Summary findings in nine governorates. Am $\mathbf{J}$. Trop Med Hyg; 62: 88-99.

[4] Arnaud, V., Li, J., Wang, Y., Fu, X., Mengzhi, S., Luo, X., et al., (2008). Regulatory role of interleukin-10 and interferon-gamma in severe hepatic central and peripheral fibrosis in humans infected with Schistosoma japonicum. J. Inf Dis; 198: 418-426.

[5] Chen, M., (2005). Use of praziquantel for clinical treatment and morbidity control of schistosomiasis japonica in China: a review of 30 years' experience. ActaTropica; 96: 168-176.

[6] Friedman, L., (2004). Controversies in liver biopsy: Who, where, when, how, why. Curr Gastroenterol Reports; 6: 30-36.

[7] Shiha, G., Sarin, S., Ibrahim, A., Omata, M., Kumar, A., Lesmana, L., et al., (2009). Liver fibrosis: Consensus recommendations of the Asian Pacific Association for the 
Study of the Liver (APASL).

HepatolInt; 3: 323-333.

[8] Li, Y., Chen, D., Ross, A., Burke, M., Yu, X., Li, R., et al., (2011). Severe hepatosplenic schistosomiasis: Clinico pathologic study of 102 cases undergoing splenectomy. Human Pathol; 42: 111-119.

[9] Hou, X., Ellis, M., McManus, D., Wang, Y., Li, S., Williams, G., et al., (2011). Diagnostic value of noninvasive bio-markers for stagespecific diagnosis of hepatic fibrosis in patients with advanced schistosomiasis japonica. Int. J. Parasitol; 41: 325-332.

[10] Wu, Y., Xu, N., Hu, J., Xu, X., Wu, W., Gao, S., et al., (2013). A simple noninvasive index to predict significant liver fibrosis in patients with advanced schistosomiasis japonica. Parasitol Int.; 62: 283-288.

[11] Agha, A., Abdulhadi, M., Marenco, S., Bella, A., Al Saudi, D., ElHaddad, A., et al., (2015). Use of the platelet count/spleen diameter ratio for the noninvasive diagnosis of esophageal varices in patients with schistosomiasis. Saudi J.

Gastroenterol; 41: 190-194.

[12] Castera, L., Foucher, J., Bernard, P., Carvalho, F., Allaix, D., Merrouche, W., et al., (2010). Pitfalls of liver stiffness measurement: A 5-year prospective study of 13,369 examinations. Hepatology; 5: 828835.

[13] Esmat, G., Elsharkawy, A., El Akel, W., Fouad, A., Helal, K., et al., (2013). Fibroscan of chronic HCV patients coinfected with schistosom- iasis. Arab J. Gastroenterol; 14:109112.

[14] Gad, A., Tanaka, E., Orii, K., Rokuhara, A., Nooman, Z., Serwah, A., et al., (2001). Relationship between hepatitis $\mathrm{C}$ virus infection and schistosomal liver disease: not simply an additive effect. $\boldsymbol{J}$. Gastroenterol; 36: 753-758.

[15] U.S. Department of Health and Human Services, National Institutes of Health, National Cancer Institute. Common Terminology Criteria for Adverse Events (CTCAE), Version 4.0, May 28, 2009 (v4.03: June 14, 2010).

[16] Castera, L., Forns, X., Alberti, A., (2008). Non-invasive evaluation of liver fibrosis using transient elastography. J. Hepatol; 48: 835-847.

[17] Castera, L., Vergniol, J., Foucher, J., Le Bail, B., Chanteloup, E., Haaser, M., et al., (2005). Prospective comparison of transient elastography, fibro test, APRI, and liver biopsy for the assessment of fibrosis in chronic hepatitis C. Gastroenterology; 128: 43-50.

[18] Lebray, P., Varnous, S., Charlotte, F., Varaut, A., Poynard, T., Ratziu, V., (2008). Liver stiffness is an unreliable marker of liver fibrosis in patients with cardiac insufficiency. Hepatology; 48: 2089

[19] Shiha, G., Zalata, K., (2001). Does schistosomiasis interfere with application of Knodell score for assessment of chronic hepatitis C? Med SciMonit; 7 (Suppl 1): 241-245.

[20] Abdel-Rahman, M., El-Sayed, M., El Raziky, M., Elsharkawy, A., El-Akel, 
W., Ghoneim, H., et al., (2013).

Coinfection with hepatitis $\mathrm{C}$ virus and schistosomiasis: Fibrosis and treatment response. World $\boldsymbol{J}$. Gastroenterol; 19: 2691-2696.

[21] Helal, T., Danial, M., Ahmed, H., (1998). The relationship between hepatitis $\mathrm{C}$ virus and schistosomiasis: histopathologic evaluation of liver biopsy specimens. Hum Pathol; 29: 743-749.

[22] Saad, Y., Said, M., Idris, M., Rabbe, M., Salama, Z., (2013). Liver stiffness measurement by FibroScan predicts the presence and size of esophageal varices in Egyptian patients with $\mathrm{HCV}$ related liver cirrhosis. J. ClinDiagnos Res; 7: 253-257.

[23] WHO, (1991). Meeting on ultrasonography in schistosomiasis: Proposal for a practical guide to the standardized use of ultrasound in the assessment of pathological changes. World Health Organization, Geneva.

[24] Richter, J., Hatz, C., Campagne, G., Bergquist, N., Jenkins, J., (2000). Ultrasound in schistosomiasis: A practical guide to the standardized use of ultrasonography for the assessment of schistosomiasis-related morbidity. World Health Organization, Geneva.

[25] Richter, J., Domingues, A., Barata, C., Prata, A., Lambertucci, J., (2001).

Report of the second satellite symposium on ultrasound in schistosomiasis. MemInstOswaldo Cruz; 96 (Suppl): 151-156.

[26] King, C., Magak, P., Salam, E., Ouma, J., Kariuki, H., Blanton, R., (2003). World Health Organization. Measuring morbidity in schistosoma mansoni: relationship between image pattern, portal vein diameter and portal branch thickness in large-scale surveys using new WHO coding guidelines for ultrasound in schistosomiasis. Trop Med Int. Health; 8: 109-117.

[27] Hoffmann, H., Esterre, P., Ravaoalimalala, V., Ehrich, J., Doehring, E., (2001). Morbidity of schistosomiasismansoni in the highlands of Madagascar and comparison of current sonographical classification systems. Trans R Soc Trop Med Hyg; 95: 623-629. 